\title{
Mössbauer spectroscopy investigation of magnetic nanoparticles incorporated into carbon nanotubes obtained by the injection CVD method
}

\author{
A.L. Prudnikava ${ }^{1}$, J.A. Fedotova ${ }^{2}$, J.V. Kasiuk ${ }^{2}$, B.G. Shulitski ${ }^{1}$, V.A. Labunov ${ }^{1}$ \\ ${ }^{1}$ Belarusian State University of Informatics and Radioelectronics, \\ P. Brovka Str. 6, Minsk 220013, Belarus; phone/fax: +375 (17) 202-10-05 \\ ${ }^{2}$ NC PHEP Belarusian State University, M. Bogdanovich Str., 153b, Minsk 220040, Belarus \\ Phone: +375 (17) 292-60-34; fax: +375(17) 292-60-75
}

\begin{abstract}
This paper is devoted to the investigation of phase composition of the magnetic filler located inside multi-wall carbon nanotubes (CNTs) using Mössbauer spectroscopy, scanning electron microscopy (SEM) and transmission electron microscopy (TEM). CNTs were obtained by the injection CVD method using ferrocenexylene solution under various conditions (i.e., the ferrocene concentration $c_{x}(1 \%, 5 \%$, and $10 \%$ ), slow or fast cooling down rates of the synthesis reactor, temperature in the reaction zone during synthesis), which can influence the magnetic properties of CNTs owing to different contribution of Fe-containing phases. SEM and TEM methods were applied to investigate morphology and structure of the synthesized material. It was shown that $\mathrm{Fe}_{3} \mathrm{C}$ phase formation is favorable at high content of the catalyst in the feeding solution $(10 \%)$, relatively low temperatures $\left(775^{\circ} \mathrm{C}\right)$ during CNTs synthesis, as well as long duration of the CNTs growth process $(5 \mathrm{~min})$. The cooling rate of the reactor after CNTs synthesis was not crucial to iron phase contribution in our experiments.
\end{abstract}

Keywords: carbon nanotubes, Mössbauer spectroscopy, cementite, injection CVD, ferrocene.

Manuscript received 23.12.09; accepted for publication 25.03.10; published online 30.04.10.

\section{Introduction}

Carbon nanotubes (CNTs) filled with magnetic materials (Magnetically Functionalized CNTs - MFCNTs) are very attractive for potential industrial applications, particularly, as an integral part of high-density magnetic storage devices, sensors for magnetic scanning probe microscopes [1], biological probes [2] or microwave irradiation shielding material [3]. A lot of researchers reported stability of the encapsulated into CNTs nanoparticles with respect to their easy oxidation and magnetostatic interactions [4].

Among numerous methods, CVD was proved to be suitable for obtaining such MFCNT arrays. Nanoparticles of $\mathrm{Fe}, \mathrm{Ni}$, Co serve as catalysts for CNTs growth and are incorporated into $\mathrm{CNT}$ s channels during the growth process. Besides, the so-called injection CVD method is commonly used under which feeding solution (mixture of fluid hydrocarbon and metallocene) is injected into the reaction zone. This method insures quite reproducible results on the MFCNTs properties. However, even this method does not give any commensurable results on the MFCNTs properties, as it was carried out under different conditions by various researchers. Therefore, for practical applications of MFCNTs it is essential to determine an accurate correlation between the synthesis conditions and their resulting consuming magnetic properties.

Actually, the results of the investigations and the conclusions concerning Fe-containing phases responsible for ferromagnetism in MFCNT arrays synthesized by the injection CVD method are quite ambiguous. It was reported that MFCNT arrays synthesized by the injection CVD method with the use of ferrocene contain nanoparticles that in general represent single crystals of $\gamma$-Fe (fcc) phase with orientation (111), $\alpha-\mathrm{Fe}$ (bcc) phase with orientation (110) or cementite $\mathrm{Fe}_{3} \mathrm{C}$ phase [4-8]. These single 
crystals can be oriented in various crystallographic planes relatively CNT walls [8]. Some researchers show that ferromagnetism is determined substantially by presence of $\gamma$-Fe phase [5], $\alpha$-Fe phase [8-11], $\mathrm{Fe}_{3} \mathrm{C}$ phase $[9,12]$, or superposition of two [10] or three [1214] mentioned phases. Previously, we observed some amount of $\mathrm{Fe}_{5} \mathrm{C}_{2}$ in our MFCNT samples by X-ray analysis in addition to $\mathrm{Fe}_{3} \mathrm{C}$ and $\alpha-\mathrm{Fe}$ [15].

One of the factors influencing the magnetic properties of MFCNTs obtained by the injection CVD method is the metallocene concentration in the feeding solution, which eventually determines the concentration of the ferromagnetic nanoparticles in the MFCNT arrays and/or different phase composition. It was determined by us that the higher is the concentration of the feeding solution, the stronger is the specific magnetization of the MFCNTs, while no additional phases were found by Xray analysis [16].

The contradictory results on the Fe-phase composition by different researchers may be caused by the factors presented below.

According to the phase diagram Fe-C [17] at $860{ }^{\circ} \mathrm{C}, \gamma$-Fe phase exists with 6.5 wt. $\%$ of carbon. This phase is thermodynamically metastable at ambient conditions. During the process of cooling to the temperatures lower than the eutectics temperature $\left(738^{\circ} \mathrm{C}\right), \gamma$-Fe phase is transformed into $\alpha$-Fe and $\mathrm{Fe}_{3} \mathrm{C}$ phases which are stable under ambient conditions. One should note that $\alpha-\mathrm{Fe}$ and $\mathrm{Fe}_{3} \mathrm{C}$ phases are ferromagnetic, but $\gamma$-Fe is antiferromagnetic $[18,19]$.

However, theoretical studies have predicted the existence of two different magnetic states in $\gamma$-Fe phase: a ferromagnetic (or high-spin) state and antiferromagnetic (or low-spin) state, what depends on the lattice parameters. In particular, one can obtain a stable at ambient conditions ferromagnetic state of $\gamma$-Fe phase by diminishing the specimen size to some monolayers or by incorporating $\mathrm{C}$ atoms into the $\gamma$-Fe lattice [20,21].

MFCNT arrays containing encapsulated $\mathrm{Fe}$ nanoparticles represent an ideal medium providing such conditions. In this case, $\mathrm{Fe}$ nanoparticles during the process of synthesis are saturated by $\mathrm{C}$ atoms, what results in the lattice expansion of $\gamma$-Fe phase. The lattice parameters almost linearly depend on the number of dissolved $\mathrm{C}$ atoms per atom of $\mathrm{Fe}$ dissolvent. Almost $10 \%$ of $\mathrm{C}$ atoms can be dissolved inside the lattice of $\gamma$ Fe phase [6]. The lattice expansion of $\gamma$-Fe phase is restrained by the strength of CNTs [22]. CNT walls create contraction force, which block $\gamma$-Fe nanoparticles ("quenching" process). Consequently, a thermodynamically stable in ambient conditions $\gamma$-Fe phase is created, which exhibits ferromagnetic properties.

The lattice expansion of $\gamma$-Fe phase owing to the incorporated $\mathrm{C}$ atoms leads to the change of its electronic structure, contributing to the stable in ambient conditions $\gamma-\mathrm{Fe}$ phase formation. First-principles calculations confirm that ferromagnetism observed in $\gamma$ $\mathrm{Fe}$ phase is connected with lattice expansion of $\gamma$-Fe phase owing to the incorporated $\mathrm{C}$ atoms and the charge transmission between $\mathrm{Fe}$ and $\mathrm{C}$ [5].

It was also claimed that the cooling rate of MFCNT arrays defines whether the "quenching" process of $\gamma$-Fe nanoparticles or its phase transformation to $\alpha-\mathrm{Fe}, \mathrm{Fe}_{3} \mathrm{C}$ phases take place [9-11]. The slower is the cooling process, the stronger the transformation probability will be [6], although in most publications there is no any reference concerning this problem.

With respect to the reported results as well as to our previous work [16], this study was focused on the effects of the ferrocene concentration in the feeding solution on Fe-containing phase contribution in the filler of the MFCNT arrays synthesized by the injection CVD method. Moreover, the influence of the cooling down rate of the reactor after MFCNT synthesis process, as well as the temperature in the reaction zone during CNTs growth, on the contribution of Fe-containing phases was also studied.

\section{Experimental}

The regimes of MFCNT array synthesis in our experiments were chosen as follows. In the first experiment ( $1^{\text {st }}$ set of samples), the ferrocene concentration $c_{x}$ in the feeding solution was $1 \%, 5 \%$ and $10 \%$, temperature in the reaction zone $T$ equaled $875^{\circ} \mathrm{C}$. The process duration time $t$ was $1 \mathrm{~min}$. The cooling rate of the synthesized MFCNT arrays was determined by the natural cooling down rate of the heating furnace. In the second experiment ( $2^{\text {nd }}$ set of samples), $c_{x}=10 \%$, $T=825^{\circ} \mathrm{C}, t=4 \mathrm{~min}$. The cooling method for this case was different: either slow realized by natural cooling down rate, or fast, using Ar stream of $450 \mathrm{~cm}^{3} \cdot \mathrm{min}^{-1}$ and immediate extracting of the CNT samples out of the reactor. In the third experiment $\left(3^{\text {rd }}\right.$ set of samples), two different temperatures for CNTs synthesis were used: $775^{\circ} \mathrm{C}$ and $875^{\circ} \mathrm{C} ; c_{x}=1 \%, t=4$ min with fast cooling down rate of the furnace. The injection rate of the feeding solution into the reaction zone $v=1 \mathrm{ml} \cdot \mathrm{min}^{-1}$ and Ar flow rate $v_{\mathrm{Ar}}=100 \mathrm{~cm}^{3} \cdot \mathrm{min}^{-1}$ were provided in all the experiments.

The morphology and structure of MFCNT arrays was investigated by scanning (SEM, Hitachi S4800) and transmission electron microscopes (TEM, JEM 100-CX, JEOL). The local configurations of iron ions of MFCNTs filler were explored by Mössbauer spectroscopy. The Mössbauer spectra were collected in transmission geometry at room temperature using the spectrometer MS2000 with ${ }^{57} \mathrm{Fe} / \mathrm{Rh}$ source $(40 \mathrm{mCu})$. Spectra were fitted by MOSMOD program assuming distribution of the hyperfine magnetic fields $H_{\text {eff }}$ and quadrupole splittings $\Delta E$ within iron nuclei. All isomer shifts are shown relatively the $\alpha$-Fe reference spectrum. During the fitting procedure, the value of the width on the half-height (FWHM) of Lorenzian line which approximates the resonance lines was fixed and equaled to $0.15 \mathrm{~mm} / \mathrm{s}$. 

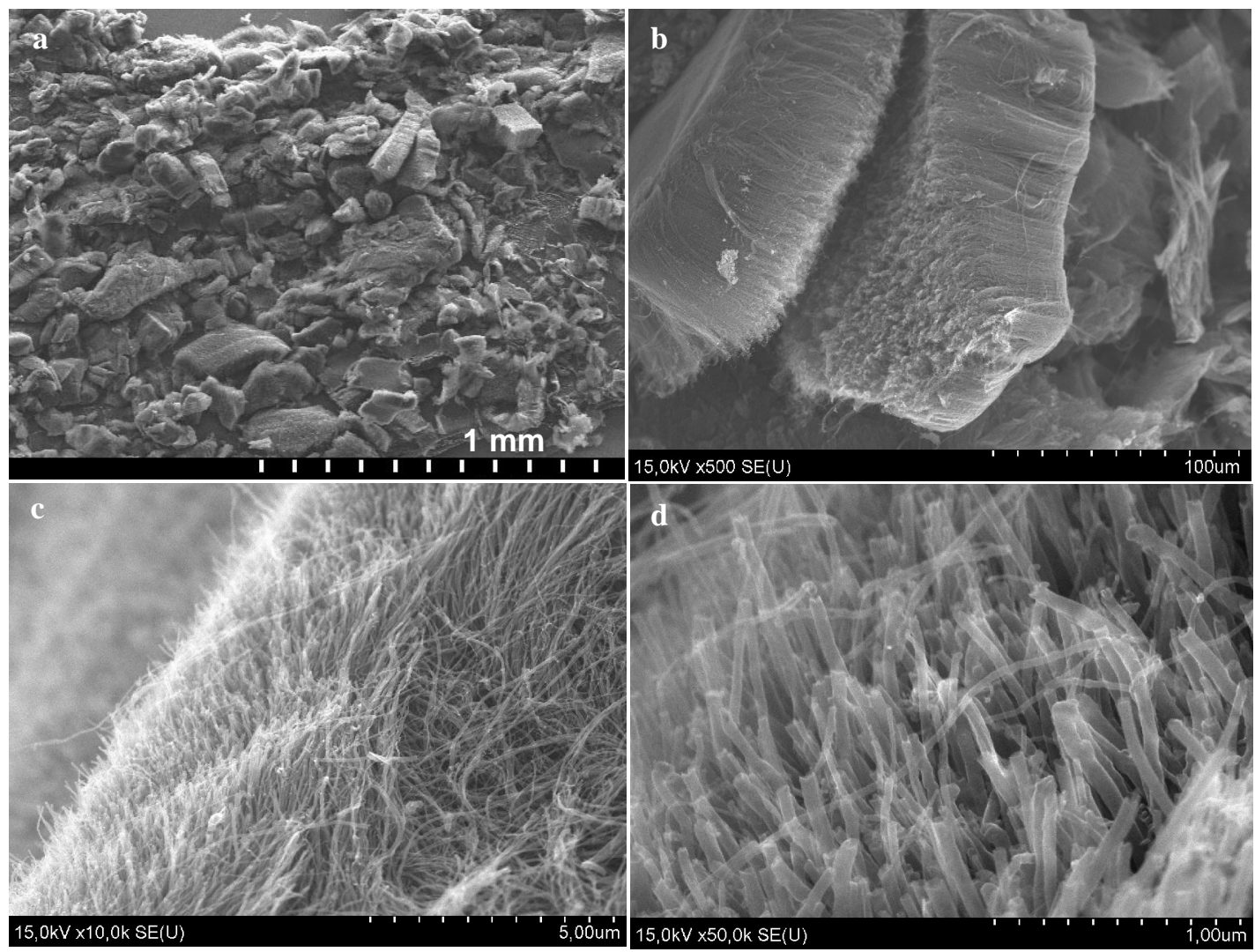

Fig. 1. SEM images of the MFCNTs scratched from silica walls of the reactor synthesized using 1\%-solution of ferrocene: $(a, b)$ general views of the powdered material placed on a scotch tape; (b, c) MFCNTs at higher magnifications

\section{Results and discussion}

For all the applied experimental regimes, the densely packed vertically aligned MFCNT arrays were formed on the walls of a silica tube of the reactor that were subsequently peeled off for the investigation in the form of powder material. Fig. 1a-d shows typical MFCNTs of the $1^{\text {st }}$ set using $1 \%$-solution of ferrocene and scratched from the quartz reactor walls. In [16] it was revealed by TEM and X-ray analysis that MFCNT for all the concentrations $c_{x}(0.55 \%, 5 \%$ and $10 \%)$ are filled with nanoparticles that are mostly represented by $\mathrm{Fe}_{3} \mathrm{C}, \alpha-\mathrm{Fe}$ and $\mathrm{Fe}_{5} \mathrm{C}_{2}$ phases.

The Mössbauer spectra of the $1^{\text {st }}$ set of MFCNTs containing three different concentrations of the feeding solution $\left(c_{x}=1 \%, 5 \%\right.$ and $\left.10 \%\right)$ are presented in Fig. 2ac, correspondingly. The best fitting of the spectra was performed in assumption of four different subspectra, i.e. singlet $C$, quadrupole doublet $D$ and two magnetic sextets $S_{1}$ and $S_{2}$. Hyperfine parameters of the subspectra are summarized in Table 1.

The parameters of singlet $C$ and sextet $S_{2}$ are characteristic of fcc $\gamma$-Fe and bcc $\alpha$-Fe, respectively. Spectra fitting confirmed (see Table 1) that $\gamma$-Fe phase existing at $860{ }^{\circ} \mathrm{C}$ in accordance with $\mathrm{Fe}-\mathrm{C}$ phase diagram was also detected during the cooling process at all $c_{x}$ and partially transformed into ferromagnetic $\alpha-\mathrm{Fe}$,
$\mathrm{Fe}_{3} \mathrm{C}$ and antiferromagnetic $\mathrm{FeC}_{2}$ phases. It is to admit that the contribution of $\gamma-\mathrm{Fe}$ nanoparticles was quite significant (between 20\% and 31\%). It means that the "quenching" process draws to the formation of the $\gamma-\mathrm{Fe}$ phase thermodynamically stable in ambient conditions. The relative contribution of $\gamma$-Fe and $\alpha$-Fe phases for $5 \%$ and $10 \%$ samples is almost the same, but for $1 \%$ it is comparatively higher. Sextet $S_{1}$ should be assigned to the cementite phase $\mathrm{Fe}_{3} \mathrm{C}$ in strong correlation with X-ray diffraction data [23]. It is important to note the presence of nonmagnetic $\mathrm{FeC}_{2}$ phase characterized with quadrupole doublet $D$. The relationship between contributions of $\mathrm{Fe}_{3} \mathrm{C}$ and $\mathrm{FeC}_{2}$ phases revealed the dependence on $c_{x}$ in these samples. In fact, $\mathrm{Fe}_{3} \mathrm{C}$ contribution increased with $c_{x}$, at the expense of $\mathrm{FeC}_{2}$ Particularly, in the applied conditions of synthesis $\mathrm{Fe}_{3} \mathrm{C}$ content grows up from $21 \%$ at $c_{x}=1 \%$ up to its higher value $54 \%$ at $c_{x}=10 \%$.

Typical TEM images of MFCNTs obtained in the second experiment ( ${ }^{\text {nd }}$ set of samples) where the method of the equipment cooling after synthesis was different, either slow or fast, are depicted in Fig. 3a-d. It is seen that there is no any visible difference between the samples. Absence of noticeable changes in the phase composition was also detected by Mössbauer investigation (Fig. 4). Hyperfine parameters extracted for the $2^{\text {nd }}$ set of samples are presented in Table 2 . 
Table 1. Hyperfine parameters of the spectra of MFCNTs synthesized with various concentrations $c_{x}$ of feeding solution ( $\left(^{\text {st }}\right.$ set of samples).

\begin{tabular}{|c|c|c|c|c|c|c|}
\hline$\partial_{0}^{0}$ & 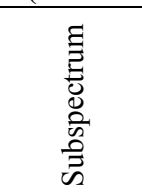 & $\begin{array}{c}{ }^{\top} \infty \\
\dot{\Xi} \\
\underbrace{\prime} \\
\infty\end{array}$ & $\begin{array}{l}T_{\infty} \\
\dot{\tilde{E}} \\
\dot{\Xi} \\
\dot{y}\end{array}$ & 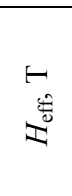 & 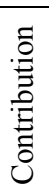 & $\begin{array}{l}0 \\
\mathbb{Z} \\
\frac{\pi}{2}\end{array}$ \\
\hline \multirow{4}{*}{1} & Singlet $C$ & -0.10 & 0 & - & 31 & $\gamma-\mathrm{Fe}$ \\
\hline & Doublet $D$ & 0.2 & 0.52 & - & 31 & $\mathrm{FeC}_{2}$ \\
\hline & Sextet $S_{1}$ & 0.17 & 0 & 20.7 & 21 & $\mathrm{Fe}_{3} \mathrm{C}$ \\
\hline & Sextet $S_{2}$ & 0.06 & -0.03 & 32.2 & 17 & $\alpha-\mathrm{Fe}$ \\
\hline \multirow{4}{*}{5} & Singlet $C$ & -0.13 & -0.11 & - & 21 & $\gamma$-Fe \\
\hline & Doublet $D$ & 0.17 & 0.48 & - & 28 & $\mathrm{FeC}_{2}$ \\
\hline & Sextet $S_{1}$ & 0.16 & 0.02 & 20.5 & 37 & $\mathrm{Fe}_{3} \mathrm{C}$ \\
\hline & Sextet $S_{2}$ & -0.05 & 0 & 32.3 & 14 & $\alpha-\mathrm{Fe}$ \\
\hline \multirow{4}{*}{10} & Singlet $C$ & -0.13 & 0 & - & 20 & $\gamma-\mathrm{Fe}$ \\
\hline & Doublet $D$ & 0.20 & 0.52 & - & 13 & $\mathrm{FeC}_{2}$ \\
\hline & Sextet $S_{1}$ & 0.17 & 0 & 20.6 & 54 & $\mathrm{Fe}_{3} \mathrm{C}$ \\
\hline & Sextet $S_{2}$ & -0.06 & -0.03 & 32.6 & 13 & $\alpha-\mathrm{Fe}$ \\
\hline
\end{tabular}

Note. $\delta$ - isomer shift, $\Delta E$ - quadrupole spitting, $H_{\text {eff }}$ - hyperfine magnetic field $\left(T=875^{\circ} \mathrm{C}, t=1 \mathrm{~min}\right.$, slow cooling rate).

According to Table 2, as in the case of Table 1, Mössbauer spectra could be fitted with four subspectra characterizing $\gamma$-Fe, $\alpha$-Fe, $\mathrm{Fe}_{3} \mathrm{C}$ and $\mathrm{FeC}_{2}$ phases. For both samples, the contribution of cementite is the largest, $\sim 82 \%$, and their hyperfine parameters coincide within the error limits. There is a little difference in contributions of $\alpha-\mathrm{Fe}$ subspectra. In the slow-cooled sample $8 \%$ of $\alpha-\mathrm{Fe}$ was formed, whereas in the fastcooled one $-6 \%$. Besides, the width of spectral line of the latter sample is larger than that of the first one, evidencing that slow cooling results in better crystallization of $\alpha-\mathrm{Fe}$.

Table 2. Hyperfine parameters of the spectra of MFCNTs after fast and slow cooling down rates.

\begin{tabular}{|c|c|c|c|c|c|c|}
\hline Cooling & Subspectrum & $\begin{array}{c}T_{\infty} \\
\dot{\Xi} \\
0^{\circ}\end{array}$ & $\begin{array}{c}T_{\infty} \\
\dot{\Xi} \\
\dot{\Xi} \\
\dot{y}\end{array}$ & $\begin{array}{c}H_{\text {eff }}, \\
\mathrm{T}\end{array}$ & $\begin{array}{l}\text { Contri- } \\
\text { bution }\end{array}$ & Phase \\
\hline \multirow{4}{*}{ fast } & Singlet $C$ & $\begin{array}{c}- \\
0.11 \\
\end{array}$ & 0 & - & 7 & $\gamma$-Fe \\
\hline & Doublet $D$ & 0.15 & 0.48 & - & 5 & $\mathrm{FeC}_{2}$ \\
\hline & Sextet $S_{1}$ & 0.18 & $\begin{array}{c}- \\
0.02\end{array}$ & 20.7 & 82 & $\mathrm{Fe}_{3} \mathrm{C}$ \\
\hline & Sextet $S_{2}$ & 0.01 & 0.01 & 33.6 & 6 & $\alpha-\mathrm{Fe}$ \\
\hline \multirow{4}{*}{ slow } & Singlet $C$ & \begin{tabular}{|c|}
- \\
0.09
\end{tabular} & 0 & - & 6 & $\gamma$-Fe \\
\hline & Doublet $D$ & 0.12 & 0.48 & - & 5 & $\mathrm{FeC}_{2}$ \\
\hline & Sextet $S_{1}$ & 0.19 & 0.01 & 20.6 & 81 & $\mathrm{Fe}_{3} \mathrm{C}$ \\
\hline & Sextet $S_{2}$ & $\begin{array}{c}- \\
0.02\end{array}$ & $\begin{array}{c}- \\
0.01\end{array}$ & 33.5 & 8 & $\alpha-\mathrm{Fe}$ \\
\hline
\end{tabular}

Note. $\delta-$ isomer shift, $\Delta E-$ quadrupole spitting, $H_{\text {eff }}-$ hyperfine magnetic field $\left(c_{x}=10 \%, T=825^{\circ} \mathrm{C}, t=4 \mathrm{~min}\right)$.

Beside the described above magnetic subspectra, two nonmagnetic components - singlet corresponding to
$\gamma$-Fe and doublet belonging to $\mathrm{FeC}_{2}-$ were detected. The doublets possess similar hyperfine parameters and almost the same relative contributions in both samples. The $\gamma$-Fe contribution was a little bit higher in the fastcooled sample (Table 2).

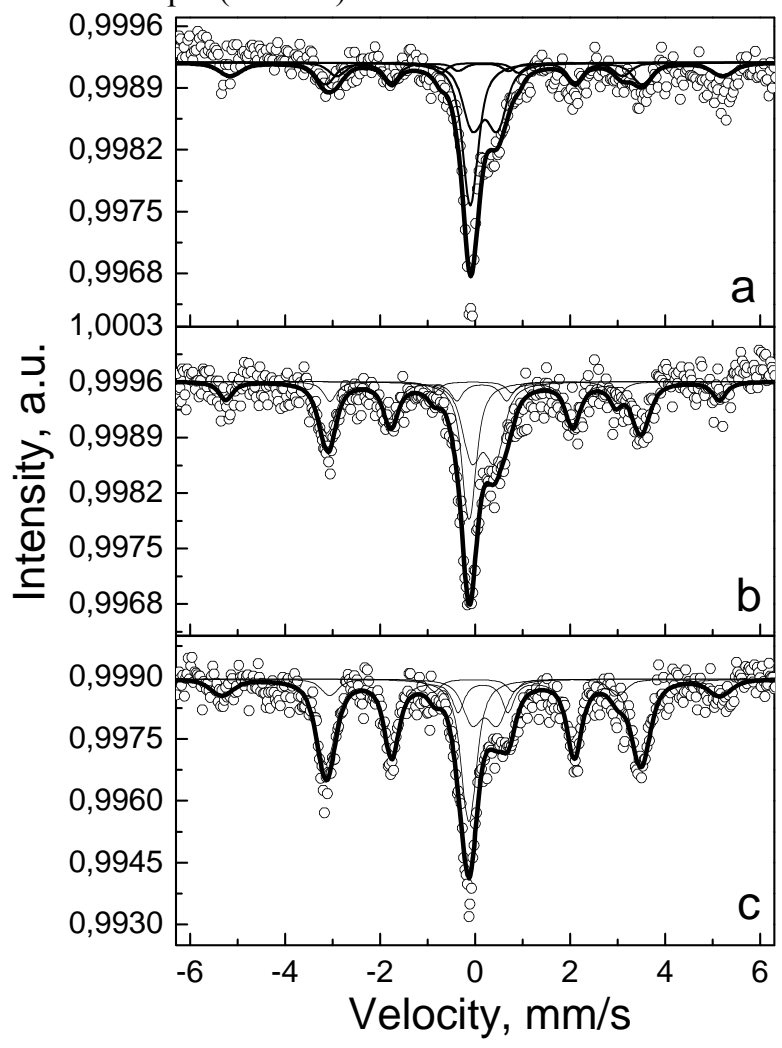

Fig. 2. Mössbauer spectra of MFCNTs synthesized at $c_{x}=$ $1 \%$ (a), $5 \%$ (b) and $10 \%$ (c) $\left(t=1 \mathrm{~min}, T=875^{\circ} \mathrm{C}\right.$, natural cooling down rate of the reactor).

As compared to our results summarized in Table 1, where the concentration of the feeding solution was the same $\left(c_{x}=10 \%\right)$, the contribution of $\mathrm{Fe}$-containing phases was different: $\mathrm{Fe}_{3} \mathrm{C} \sim 80 \%$ instead of $\sim 50 \%$ (see Table 1). In our previous paper [23], the content of cementite phase was even higher $(\sim 90 \%)$. The specific feature of these experiments is the duration of CNT synthesis process: $1 \mathrm{~min}$ (for the $1^{\text {st }}$ set of samples, see Table 1); 4 min (for the $2^{\text {nd }}$ set of samples, see Table 2), and $5 \mathrm{~min}$ for the samples considered in [23]. Therefore, the duration of the synthesis process strongly influences the contribution of iron-containing phases, i.e. the higher is the duration, the higher the content of $\mathrm{Fe}_{3} \mathrm{C}$ phase will be. It is obvious that for obtaining the maximum amount of $\mathrm{Fe}_{3} \mathrm{C}$ phase, the process duration of $5 \mathrm{~min}$ is optimal in our experiments. According to [5], in the case when the process duration exceeds $20 \mathrm{~min}$, the annealing of $\mathrm{Fe}$ is going on, which can lead to decomposition of even thermodynamically stable $\mathrm{Fe}_{3} \mathrm{C}$. CNTs destruction and its morphology deterioration take place. External and internal diameters of CNTs and distance between them are enlarged up to $50 \%$, branching is appearing and number of spherical shape Fe particles is increasing. 


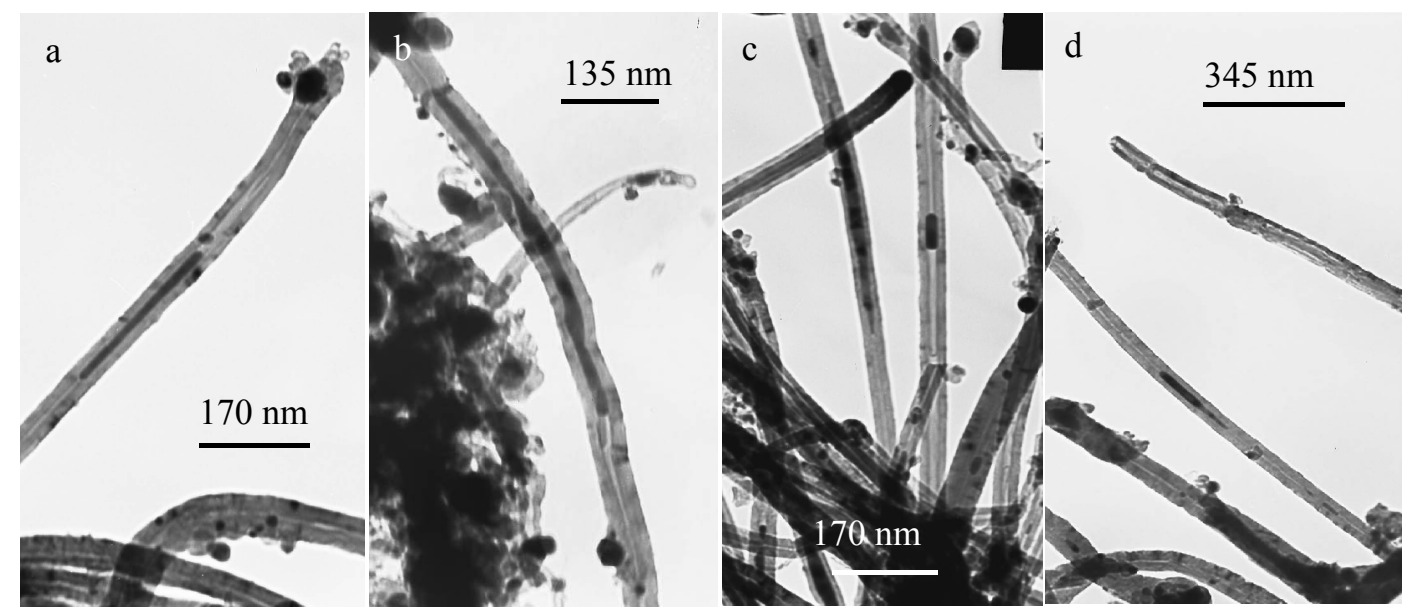

Fig. 3. Typical TEM images of MFCNTs after different cooling down rates of the synthesis equipment: (a, b) fast; (c, d) slow.
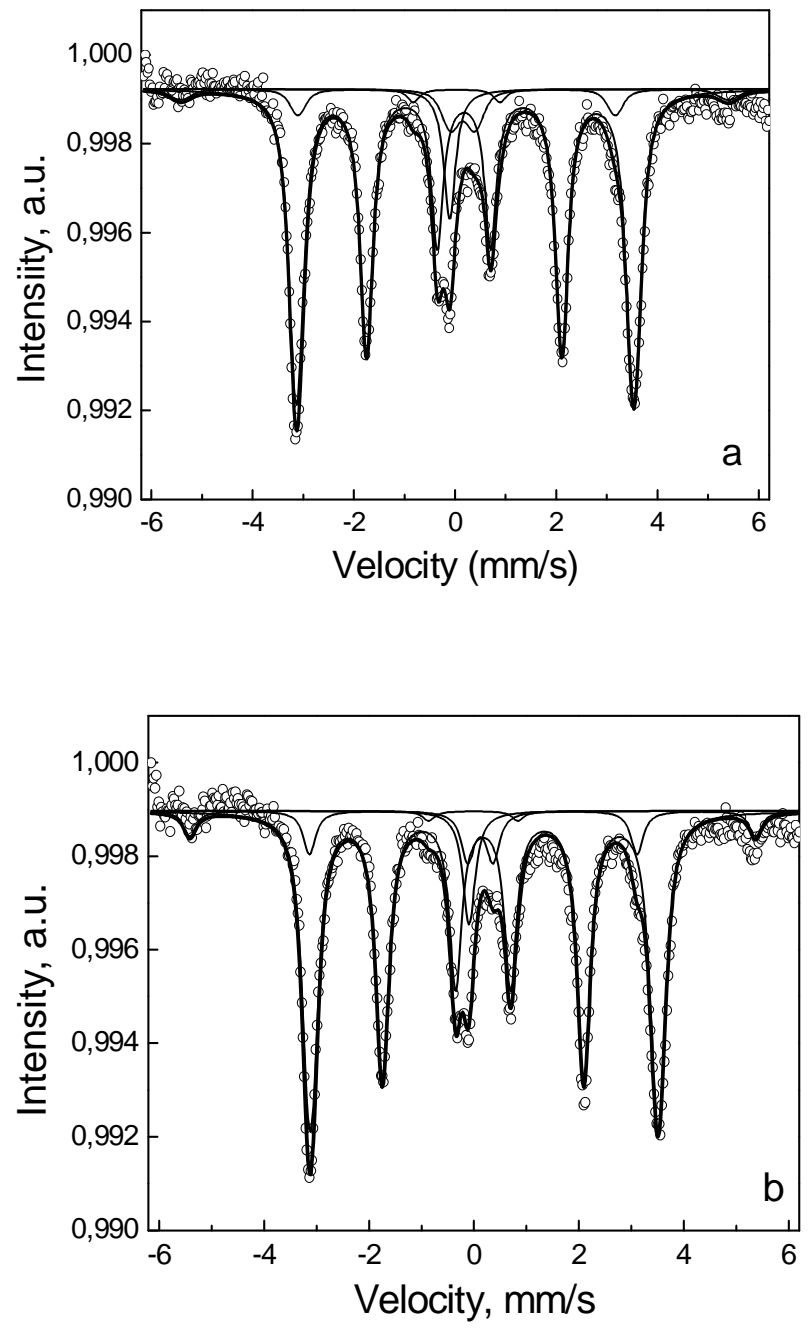

Fig. 4. Mössbauer spectra of MFCNTs synthesized at $c_{x}=10 \%, t=3.5 \mathrm{~min}, T=825^{\circ} \mathrm{C}$ after fast (a) and slow (b) cooling down rates of the synthesis equipment.
Table 3. Hyperfine parameters of the spectra of MFCNTs synthesized at $775^{\circ} \mathrm{C}$ and $875^{\circ} \mathrm{C}$.

\begin{tabular}{|c|c|c|c|c|c|c|}
\hline 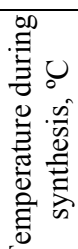 & 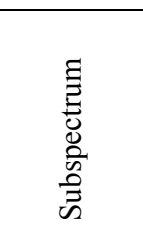 & $\begin{array}{c}T_{\infty} \\
\dot{\Xi} \\
\dot{\Xi} \\
0^{\circ}\end{array}$ & $\begin{array}{l}T_{0}^{0} \\
\dot{\Xi} \\
\dot{\Xi} \\
\dot{y}\end{array}$ & 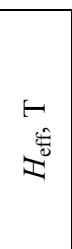 & 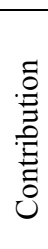 & $\begin{array}{l}\tilde{D} \\
\tilde{E} \\
\stackrel{\vec{Z}}{2}\end{array}$ \\
\hline \multirow{4}{*}{875} & Singlet $C$ & -0.10 & 0 & - & 31 & $\gamma$-Fe \\
\hline & Doublet $D$ & 0.2 & 0.52 & - & 31 & $\mathrm{FeC}_{2}$ \\
\hline & Sextet $S_{1}$ & 0.17 & 0 & 20.7 & 21 & $\mathrm{Fe}_{3} \mathrm{C}$ \\
\hline & Sextet $S_{2}$ & 0.06 & -0.03 & 32.2 & 17 & $\alpha-\mathrm{Fe}$ \\
\hline \multirow{3}{*}{775} & Doublet $D$ & 0.17 & 0.50 & - & 36 & $\mathrm{FeC}_{2}$ \\
\hline & Sextet $S_{1}$ & 0.18 & 0.02 & 20.8 & 60 & $\mathrm{Fe}_{3} \mathrm{C}$ \\
\hline & Sextet $S_{2}$ & 0.09 & 0.15 & 33.9 & 4 & $\alpha-\mathrm{Fe}$ \\
\hline
\end{tabular}

Note. $\delta-$ isomer shift, $\Delta E-$ quadrupole spitting, $H_{\text {eff }}-$ hyperfine magnetic field ( $c_{x}=0.5 \%, t=4 \mathrm{~min}$, fast cooling).

The Mössbauer spectra of the MFCNTs synthesized at two different temperatures, $775^{\circ} \mathrm{C}$ and $875^{\circ} \mathrm{C}$, using $c_{x}=1 \% ; t=4 \mathrm{~min}$ and at fast cooling of the furnace after their synthesis are shown in Fig. 5.

It is noteworthy that TEM investigations (not shown) did not reveal any new peculiarities in a way the magnetic particles fill nanotubes. Mössbauer spectra of the $3^{\text {rd }}$ set of samples revealed very different phase contributions of Fe-containing phases for two applied temperatures of synthesis. As evidenced from Table 3 summarizing the hyperfine parameters of the corresponding spectra at $T=775^{\circ} \mathrm{C}, \gamma$-Fe phase was not detected, which is in agreement with the phase diagram of $\mathrm{Fe}-\mathrm{C}$. The content of $\mathrm{Fe}_{3} \mathrm{C}$ at this temperature is $\sim 60 \%$, even higher than at $c_{x}=10 \%$ with $t=1 \mathrm{~min}$ (Table 1). One should note that at $T=775^{\circ} \mathrm{C}$ there is a very low content of $\alpha$-Fe phase (only 4\%), and contribution of $\mathrm{Fe}$ carbides definitely dominates. It means that at this relatively low temperature the process of $\gamma$-Fe phase transformation into other $\mathrm{Fe}$-containing 
phases was not detected, and $\alpha-\mathrm{Fe}, \mathrm{Fe}_{3} \mathrm{C}$ and $\mathrm{FeC}_{2}$ phases are directly created during the synthesis process. A large amount of carbides testifies their stability as compared to $\alpha$-Fe.
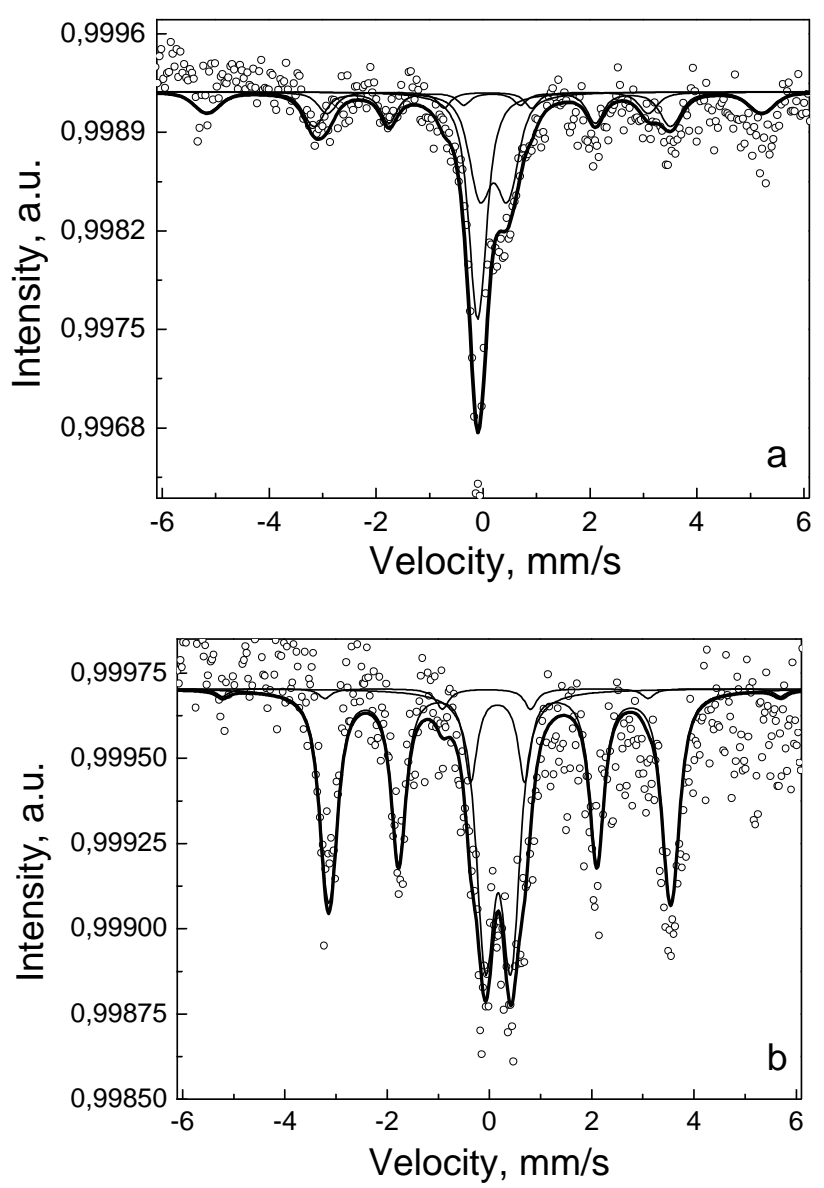

Fig. 5. Mössbauer spectra of the MFCNTs synthesized at two different temperatures, $875^{\circ} \mathrm{C}$ and $775^{\circ} \mathrm{C}\left(c_{x}=1 \%, t=4 \mathrm{~min}\right.$, fast cooling down rate of the furnace).

\section{Conclusion}

Being based on these results, one can conclude that the relatively low temperatures $\left(775^{\circ} \mathrm{C}\right)$ during $\mathrm{CNTs}$ synthesis, comparatively long duration of the CNTs growth process, as well as high content of the catalyst in the feeding solution are important factors promoting $\mathrm{Fe}_{3} \mathrm{C}$ phase formation. Formation of iron-containing phases inside carbon nanotubes appeared not to be so sensitive to the cooling down rate of the synthesis reactor, unlike it was claimed in other publications [9-11].

\section{References}

1. H. Kuramochi, H. Akinaga, Y. Semba, N. Kijima, T. Uzumaki, M. Yasutake, A. Tanaka, and H. Yokoyama, CoFe-coated carbon nanotube probes for magnetic force microscope // Jpn. J. Appl. Phys. 44, p. 2077-2080 (2005).

2. J.R. Freedman, D. Mattia, G. Korneva, Y. Gogotsi, G. Friedman and A.K. Fontecchio, Magnetically assembled carbon nanotube tipped pipettes // Appl. Phys. Lett. 90, 103108 (2007); DOI: 10.1063/1.2711521.

3. H.M. Kim, K. Kim, C.Y. Lee, and J. Joo, S.J. Cho, H.S. Yoon, D.A. Pejakovic', J.W. Yoo, and A.J. Epstein, Electrical conductivity and electromagnetic interference shielding of multiwalled carbon nanotube composites containing Fe catalyst // Appl. Phys. Lett. 84 (4), p. 589-591 (2004).

4. T. Mühl, M. Ritschel, R. Kozhuharova, D. Elefant, A. Graff, A. Leonhardt, I. Mönch, C.M. Schneider, S. Groudeva-Zotova, Magnetic properties of aligned ferromagnetically filled carbon nanotubes // Highlights 2002, p. 27-30 (2002).

5. B. Wei, M. Shima, R. Pati, S.K. Nayak, D.J. Singh, R. Ma, Y. Li, Y. Bando, S. Nasu, and P.M. Ajayan et al., Room-temperature ferromagnetism in doped face-centered cubic Fe nanoparticles // Small 2, p. 804-809 (2006).

6. N. Koprinarov, M. Konstantinova, T. Ruskov, I. Spirov, M. Marinov, Ts. Tsacheva, Ferromagnetic nanomaterials obtained by thermal decomposition of ferrocene in a closed chamber // Bulg. J. Phys. 34, p. 17-32 (2007).

7. F. Geng, H. Cong, Fe-filled carbon nanotube array with high coercivity // Physica B 382, p. 300-304 (2006).

8. C. Mueller, S. Hampel, D. Elefant, K. Biedermann, A. Leonhardt, M. Ritschel, B. Buechner, Iron filled carbon nanotubes grown on substrates with thin metal layers and their magnetic properties // Carbon 44, p. 1746-1753 (2006).

9. X.X. Zhang, G.H. Wen, S.M. Huang, L.M. Dai, R.P. Gao, Z.L. Wang, Magnetic properties of $\mathrm{Fe}$ nanoparticles trapped at the tips of the aligned carbon nanotubes // J. Magn. Magn. Mater. 231, p. L9-L12 (2001).

10. J. Jiao, S. Seraphin, X. Wang, J.C. Whiters, Solution route to single crystalline dendritic cobalt nanostructures coated with carbon shells // J. Appl. Phys. 80, p. 103-108 (1996).

11. N. Grobert, W.K. Hsu, Y.Q. Zhu, J.P. Hare, H.W. Kroto, D.R.M. Walton, M. Terrones, H. Terrones, P. Redlich, M. Ruhle, R. Escudero, F. Morales, Encapsulation, compensation, and substitution of catalyst particles during continuous growth of carbon nanotubes // Appl. Phys. Lett. 75, p. 3363-3365 (1999).

12. X.Q. Zhao, B.X. Liu, Y. Liang, Z.Q. Hu, Structure and magnetic properties of the oxide layers on iron ultrafine particles // Appl. Phys. A 64, p. 483-486 (1997).

13. H. Hofmeister, F. Huisken, B. Kohn, R. Alexandrescu, S. Cojocaru, A. Crunteanu, I. Morjan, L. Diamandescu, Filamentary iron nanostructures from laser-induced pyrolysis of iron pentacarbonyll and ethylene mixtures // Appl. Phys. $A$ 72, p. 7-11 (2001). 
14. C.N.R. Rao, A. Covindaraj, Carbon nanotubes from organometallic precursors // Acc. Chem. Res. 35, p. 998-1007 (2002); DOI: 10.1021/ar0101584.

15. V.A. Labunov, B.G. Shulitski, E.L. Prudnikava, K.I. Yanushkevitch, Structure, composition and magnetic properties of carbon nanotubes doped by Fe during the growth // J. Phys.: Conference Series 100, 052095 (2008).

16. A.L. Prudnikava, V.A. Labunov, B.G. Shulitski, K.I. Yanushkevich, A.L. Daniljuk, O.F. Demidenko, Magnetic properties of CNT arrays synthesized by the injection CVD method at various catalyst concentrations // Proc. SPIE, $12^{\text {th }}$ International workshop "Nano-Design Technology and Computer Simulations", edited by A.I. Melker, V.V. Nelayev, 7377, 73770Q (2009); DOI: 10.1117/12.836974.

17. B.C. Satishkumar, A. Govindaraj, R. Sen, C.N.R. Rao, Single-walled nanotubes by the pyrolysis of acetylene-organometallic mixtures // Chem. Phys. Lett. 293, p. 47-52 (1998).

18. H. Kim, W. Sigmund, Iron nanoparticles in carbon nanotubes at various temperatures // J. Cryst. Growth 276, p. 594-605 (2005).
19. I. Ando, X. Zhao, T. Sugai, M. Kumar, Growing carbon nanotubes // Mater. Today 7(10), p. 22-29 (2004).

20. A. Kirilyuk, J. Giergiel, J. Shen, M. Straub, J. Kirschner, Growth of stabilized $\gamma$-Fe films and their magnetic properties // Phys. Rev. B 54(2), p. 1050-1063 (1996).

21. M. Stampanoni, A. Vaterlaus, M. Aeschlimann, F. Mejer, Magnetic properties of thin fcc iron films on $\mathrm{Cu}(001)$ // J. Appl. Phys. 64, p. 5321-5324 (1988).

22. C.W. Jung, P. Jacobs, Physical and chemical properties of superparamagnetic iron oxide MR contrast agents: Ferumoxides, ferumoxtran, ferumoxsil // Magn. Res. Imaging 13(5), p. 661 (1995).

23. A.S. Basaev, B.B. Bokhonov, O.F. Demidenko, V.A. Labunov, G.I. Makovetskii, E.L. Prudnikova, A.A. Reznev, A.N. Saurov, V.M. Fedosyuk, Yu.A. Fedotova, B.G. Shulitskii, K.I. Yanushkevich, Synthesis and properties of magnetically functionalized carbon nanotubes // Nanotechnologies in Russia 3, p. 184-190 (2008). 